\title{
Whole-grain consumption and its effects on hepatic steatosis and liver enzymes in patients with non-alcoholic fatty liver disease: a randomised controlled clinical trial
}

\author{
Masoumeh Dorosti ${ }^{1}$, Ali Jafary Heidarloo ${ }^{2}$, Farnush Bakhshimoghaddam ${ }^{1}$ and Mohammad Alizadeh ${ }^{3,4 *}$ \\ ${ }^{1}$ Student Research Committee, Department of Nutrition, Faculty of Medicine, Urmia University of Medical Sciences, Urmia, Iran \\ ${ }^{2}$ Gastroenterology and Hepatology subdivision of Internal Medicine, Imam Khomeini Hospital, Faculty of Medicine, \\ Urmia University of Medical Sciences, Urmia, Iran \\ ${ }^{3}$ Food and Beverages Safety Research Center, Urmia University of Medical Sciences, Urmia, Iran \\ ${ }^{4}$ Department of Nutrition, Faculty of Medicine, Urmia University of Medical Sciences, Urmia, Iran
}

(Submitted 9 May 2019 - Final revision received 10 October 2019 - Accepted 23 October 2019 - First published online 5 November 2019)

\begin{abstract}
Non-alcoholic fatty liver disease (NAFLD) is a considerable challenge to public health across the globe. Whole grain is highly recommended as an inseparable part of a healthy diet and has been proposed as an effective way to manage NAFLD. The objective in the present study was to evaluate the effects of whole-grain consumption on hepatic steatosis and liver enzymes as primary outcomes in patients with NAFLD. Over the 12 weeks of this open-label, randomised controlled clinical trial, 112 patients (mean age 43 (sD $8 \cdot 7$ ) years; $\left.\mathrm{BMI} 32 \cdot 2(\mathrm{sD} 4 \cdot 3) \mathrm{kg} / \mathrm{m}^{2}\right) \mathrm{were}$ randomly assigned to two groups to receive dietary advice, either to obtain at least half of their cereal servings each day from whole-grain foods or from usual cereals. By the end of the study, the grades of NAFLD showed a significant decrease in the intervention group $(P<0 \cdot 001)$. In addition, a significant reduction in serum concentration of alanine aminotransferase $(P<0 \cdot 001)$, aspartate aminotransferase $(P<0 \cdot 001)$, $\gamma$-glutamyltransferase $(P=0.009)$, systolic blood pressure $(P=0.004)$ and diastolic blood pressure $(P=0.008)$ was observed in the intervention group compared with the control group. After adjusting, however, no significant differences were found between the two groups in terms of lipid profile, glycaemic status and anthropometric measurements. Overall, our study demonstrated that consumption of whole grains for 12 weeks had beneficial effects on hepatic steatosis and liver enzymes concentrations in patients with NAFLD.
\end{abstract}

Key words: Non-alcoholic fatty liver disease: Whole grains: Liver enzymes: Insulin resistance: Lipid profile

Non-alcoholic fatty liver disease (NAFLD) is the most common chronic liver disease in Western and Asian countries ${ }^{(1)}$. A metaanalysis reported that the prevalence of NAFLD in Iran was $33.95 \%$ and that it was increasing at an alarming rate ${ }^{(2)}$. It has been estimated that NAFLD will be a major cause of liver-related morbidity and mortality by $2030^{(3)}$. NAFLD describes a common clinic-pathological condition characterised by a large number of fatty deposits in hepatocytes, and it is detected by either histology or imaging ${ }^{(4)}$. NAFLD has been linked to elevation of aminotransferases, dyslipidaemia, insulin resistance, hypertension and obesity ${ }^{(5,6)}$. Moreover, NAFLD in the hepatic steatosis stage can be reversed by lifestyle modification such as weight loss, regular physical activity and adherence to a healthy $\operatorname{diet}^{(7)}$.

Whole grains are highly recommended as the inseparable part of a healthy diet, and their significance in chronic disease management is interesting. According to the definition proposed by American Association of Cereal Chemists, whole grains are the intact, ground, cracked or flaked kernel of grains, whose principal anatomical components are the starchy endosperm, germ and bran $^{(8)}$. The 2012 Dietary Guidelines for Americans recommend consuming at least half of the daily grain servings as whole grains with gram recommendations ranging from $48 \mathrm{~g} / \mathrm{d}$ to $75 \mathrm{~g} / 8368 \mathrm{~kJ}$ to reduce the risk of chronic diseases ${ }^{(9)}$.

Previous studies carried out on animal models suggested that dietary whole grains may have protective effects against chronic diseases ${ }^{(10)}$. Furthermore, it has been claimed that consumption of whole-grain products may facilitate management of metabolic disorders ${ }^{(11)}$. However, the results of a whole-grain-based case-control study found an inverse association between whole-grain consumption and NAFLD ${ }^{(12)}$. On the other hand, a large cohort study showed a negative significant correlation between whole-grain intake and

Abbreviations: MET, metabolic equivalent of task; NAFLD, non-alcoholic fatty liver disease; WHR, waist to hip circumference ratio.

* Corresponding author: Mohammad Alizadeh, fax +984432780800, email alizade85@yahoo.com, alizadeh.m@umsu.ac.ir 
hyperlipidaemia and insulin resistance ${ }^{(13)}$. Similarly, some previous studies have suggested that whole-grain foods may improve glucose and lipid biomarkers in healthy obese or hyperglycaemic adults ${ }^{(14)}$. Many randomised controlled trials have also evaluated the effects of whole-grain products on blood glucose concentrations and serum lipids, but they have found inconsistent results ${ }^{(15-18)}$.

Despite acknowledgement of the importance of whole grains in the diet by researchers, there is little evidence regarding the association between consumption of whole grains and management of NAFLD-related diseases ${ }^{(19)}$. Thus, it is hypothesised in the present study that dietary whole grains might be effective in the management of NAFLD. To evaluate this hypothesis, this randomised controlled clinical trial was designed to assess whether consumption of whole grains is effective in the management of NAFLD in adults or not.

\section{Materials and methods}

\section{Recruitment and eligibility screening}

A total of 202 adult patients diagnosed with NAFLD, who had visited Ghods Clinic in Urmia, Iran, between March and May 2017, were enrolled in the study, consecutively. NAFLD diagnosis was based on the presence of ultrasonographic evidence of fatty liver and no other type of liver injury or steatosis. All patients with NAFLD were asked to participate and were recruited, provided they met the inclusion criteria and accepted to participate in the study. The reason behind this was to eliminate any bias in patient selection. Men and women at the age of fertility, that is, 18 years or older, with fatty liver grades 1,2 or 3 and $\mathrm{BMI} \leq 40$ $\mathrm{kg} / \mathrm{m}^{2}$ were included in the study. Excluding factors consisted of a history of alcohol abuse; evidence of any acute and chronic gastrointestinal diseases other than fatty liver; autoimmune disease; type 1 or 2 diabetes; uncontrolled thyroid conditions; psychiatric disorders; impaired renal function; use of drugs to treat NAFLD; use of drugs having the potential to affect glucose and lipid metabolism during the study and over the last 6 months; history of using medications that increase the risk of NAFLD, such as perhexiline, methotrexate, amiodarone, diltiazem, tamoxifen, nifedipine, oestrogens, chloroquine and other possible hepatotoxic agents and pregnancy and breast-feeding for women. Participants who took at least half of their daily grain servings as whole grains before the start of the study were also excluded from the study.

\section{Study design}

This parallel, open-label randomised controlled clinical trial was conducted from June to August 2017. Written informed consents were obtained from all participants after they became informed about the aims and procedures of the study. The study protocol was approved by the Ethics Committee at Urmia University of Medical Sciences and was registered at the Iranian Registry of Clinical Trials website (IRCT20170206032417N3). Randomisation lists were computer-generated by a statistician, and the laboratory staff, radiologist and statistician were blinded to the intervention assignment until the end of the trial.

\section{Intervention}

At baseline, patients were randomly allocated to two groups to receive dietary advice either to obtain at least half of their cereal servings each day from whole-grain foods (intervention group $(n 56)$ ) or from usual cereals (control group ( $n$ 56)) for 12 weeks. This time period was designated based on those previous studies showing beneficial effects of whole grains on metabolic markers in 6 and 8 weeks ${ }^{(16,17)}$. A dietitian met with participants at baseline to explain the 2012 Dietary Guidelines for Americans ${ }^{(9)}$ used in the study and also to provide educational information to facilitate their understanding and adherence. Participants in the intervention group were given a list and description of whole-grain foods and were advised to consume at least half of the daily grain servings as whole grains based on recommendations in the 2012 Dietary Guidelines for Americans $^{(9)}$. In addition, participants in both groups were asked to eat, two to three servings of low-fat dairy products, five servings of fruits and vegetables and two servings of lean meat, poultry or fish on a daily basis, as recommended in the 2012 Dietary Guidelines for Americans ${ }^{(9)}$. At baseline, patients were requested to maintain their levels of physical activity throughout the trial period. Compliance with the guidelines was determined every week by a telephone call and dietary assessment on four occasions (at weeks 0, 4, 8 and 12) during the study.

\section{Liver ultrasonography}

All participants underwent a liver ultrasonographic examination performed by one operator and one expert radiologist (intra-inter-observer variation approximately 20 and $25 \%$, respectively) at baseline and at the end of the study. NAFLD was defined as the presence of an ultrasonographic pattern according to the following criteria: liver-kidney echo discrepancy, attenuated echo penetration, visibility of diaphragm and narrowing of the lumen of the hepatic veins on ultrasonography (Philips Affiniti 50 Ultrasound). Fatty liver was graded as normal, grades 1, 2 and 3, according to the modified criteria of Kurtz et $a$. $^{(20)}$.

\section{Biochemical measurements}

Biochemical testing was performed at weeks 0 and 12 to determine serum alanine aminotransferase, aspartate aminotransferase, $\gamma$-glutamyltransferase, total cholesterol, TAG, HDL-cholesterol, LDL-cholesterol, fasting blood sugar and insulin concentrations using routine automated assay methods at the Urmia University of Medical Sciences reference laboratory after an overnight fast. Serum concentrations of alanine aminotransferase, aspartate aminotransferase, ALP, total cholesterol, TAG, HDL-cholesterol, LDL-cholesterol and fasting blood sugar were measured using routine enzymatic assays with commercial kits (Pars Azmoon) and an automatic biochemical analyser (BT 4500, Biotechnica). Furthermore, serum concentrations of insulin were measured by the electrochemiluminescence method on the Cobas E411 analyser (Roche Diagnostics $\mathrm{GmbH}$ ). Homeostatic model assessment of insulin 
resistance (HOMA-IR) and quantitative insulin sensitivity check index were also calculated based on the suggested formulas ${ }^{(21)}$.

\section{Blood pressure and anthropometric measurements}

At weeks 0 and 12, systolic blood pressure and diastolic blood pressure were taken by the same person, during the same period of the day, and after $5 \mathrm{~min}$ of rest in the sitting position employing a validated mercury sphygmomanometer device (MicrolifeBP AG1-10). Furthermore, anthropometric measures were taken in the standing position, with participants wearing light clothing and no shoes, at baseline and week 12. BMI was calculated as weight in kilograms divided by height in metres squared $\left(\mathrm{kg} / \mathrm{m}^{2}\right)$; height and weight were measured using a digital scale and stadiometer (BSM370; Inbody Co.). Moreover, waist and hip circumferences were measured following recommendations by the $\mathrm{WHO}^{(22)}$. The waist to hip circumference ratios (WHR) were calculated as well.

\section{Dietary intake and physical activity assessment}

At baseline and every 4 weeks, 3-d (one weekend day and two nonconsecutive week days) 24-h diet recall interview was performed by a trained dietitian. The dietary recalls were analysed using the Nutritionist 4 (N4) software (First Databank, Inc.; Hearst Corporation) modified for Iranian foods. The obtained data were then used to estimate the mean of consumed dairy products, vegetables, fruits, cereals, whole grains, meat and fat group servings. If a whole-grain food did not exist in the N4 software, it would be replaced with a proper whole grain with similar micro- and macronutrient and dietary fibre contents. According to the 2012 dietary guidelines for Americans, grain servings were defined as $1 / 2 \mathrm{cup}(120 \mathrm{ml})$ of cooked rice, pasta or cereal; one slice of bread (30 g) and one ounce $(28 \mathrm{~g})$ of ready-to-eat cereals such as puffed products, biscuits, shred products and other conventional cereal piece shapes. Physical activities were investigated using the metabolic equivalent of task (MET) questionnaire ${ }^{(23)}$ at weeks 0,6 and 12.

\section{Primary and secondary outcomes}

The primary outcome measures were changes in liver steatosis grades and serum concentrations of liver enzymes. Secondary outcome measures were changes in serum concentrations of lipid profiles, glycaemic variables, blood pressure and anthropometric variables.

\section{Statistical analysis}

The obtained data were analysed using SPSS software version 21 (SPSS, Inc.). For all analyses, a $P$ value $<0.05$ was considered to be significant. Continuous and categorical data were presented as mean values and standard deviation and frequency, respectively. To calculate the sample size, the standard formula suggested for parallel clinical trials was applied, considering type 1 error $(\alpha)$ of 0.05 and type 2 error $(\beta)$ of 0.10 (power $=90 \%$ ) (based on a study conducted by Katcher et al. ${ }^{(24)}$ and a mean change in the total cholesterol). Based on this, the sample size was estimated to be forty-seven per study group. Assuming $19 \%$ dropouts from each group, the final sample size was estimated to be fifty-six per group. Differences in general characteristics between groups at baseline were compared by applying independent-samples Student's $t$ tests (for continuous variables) and $\chi^{2}$ (for categorical variables). Marginal model and generalised estimating equations with a cumulative logit link function were used to compare changes in the fatty liver grade among groups during the intervention. To assess confounders, the changes in fatty liver grade for baseline value of the outcome, mean change in food groups, MET value, energy intake, weight, BMI and WHR were adjusted by using the generalised estimating equations models. To identify within-group differences, pairedsamples $t$ tests were used. Repeated-measures ANOVA, using the general linear model with group (intervention and control) as the between-subjects factor, was conducted to identify changes in dietary intakes and serum concentrations of liver enzymes, lipid profile, glycaemic variables, blood pressure over time and also differences between the groups. Moreover, to assess confounders, all analyses for baseline value of the outcome, mean change in food groups, MET value, energy intake, weight, BMI and WHR were adjusted by applying the ANCOVA test.

\section{Results}

A total of 112 participants were randomly assigned to two groups. After randomisation, eighteen participants were excluded, due to failure to follow-up. In total, ninety-four participants completed the trial: the intervention group $(n 47)$ and control group ( $n$ 47) (Fig. 1). No significant differences were found between the two groups regarding baseline demographic characteristics (Table 1). On the basis of 3-d 24-h dietary recalls obtained at study baseline and throughout the intervention, significant changes between the groups, in terms of energy intake and consumed food groups, with the exception of dairy and fruit intakes were observed (Table 2).

\section{Primary outcomes}

Ultrasound grades decreased significantly in the intervention group compared with the control group $(P<0.001)$. In the intervention group, $66 \%$ of patients showed a reduction in grade of fatty liver (Table 3).

Changes in serum concentrations of alanine aminotransferase $(P<0.001)$, aspartate aminotransferase $(P<0.001)$ and $\gamma$-glutamyltransferase $(P=0.009)$ were significantly different between the two groups. The intervention group showed a greater reduction in serum concentrations of these enzymes than did the control group (Table 4). These significant differences between the groups were observed even after adjustments were made to the baseline value of the outcome, mean changes in food groups and MET value, and after more adjustments were made to the mean changes in energy intake, weight, BMI and WHR (Table 5).

\section{Secondary outcomes}

Changes in serum concentrations of total cholesterol $(P=0.004)$, LDL-cholesterol $(P=0.014)$, systolic blood pressure $(P=0.004)$ and diastolic blood pressure $(P=0.008)$ were significantly 
Table 1. Demographic characteristic of patients with non-alcoholic fatty liver disease ( $n$ 47) (Mean values and standard deviations)

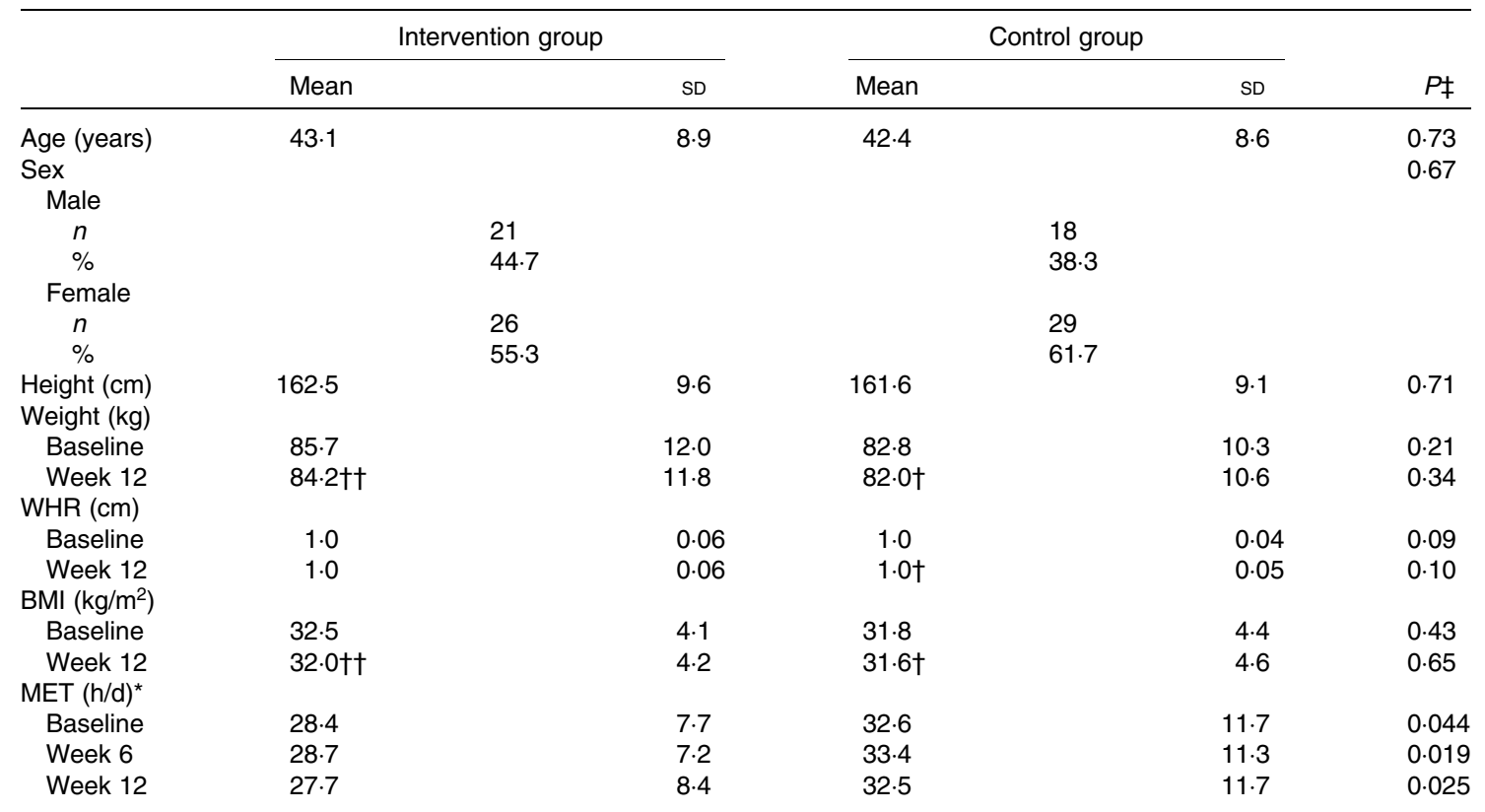

WHR, waist to hip circumference ratio; MET, metabolic equivalent of task.

${ }^{*} P$ values were computed by analysis of general linear model ANOVA for repeated measurements: $P_{\text {Time }}=0.10, P_{\text {Group }}=0.024, P_{\text {Time }} \times$ Group $=0.21$. $P$ values were computed by paired-samples $t$ test: $\dagger P<0.05$, $\dagger \dagger P<0.001$.

$\ddagger P$ values were computed by the independent-samples $t$ test for continuous variables and by $\chi^{2}$ for categorical variables.

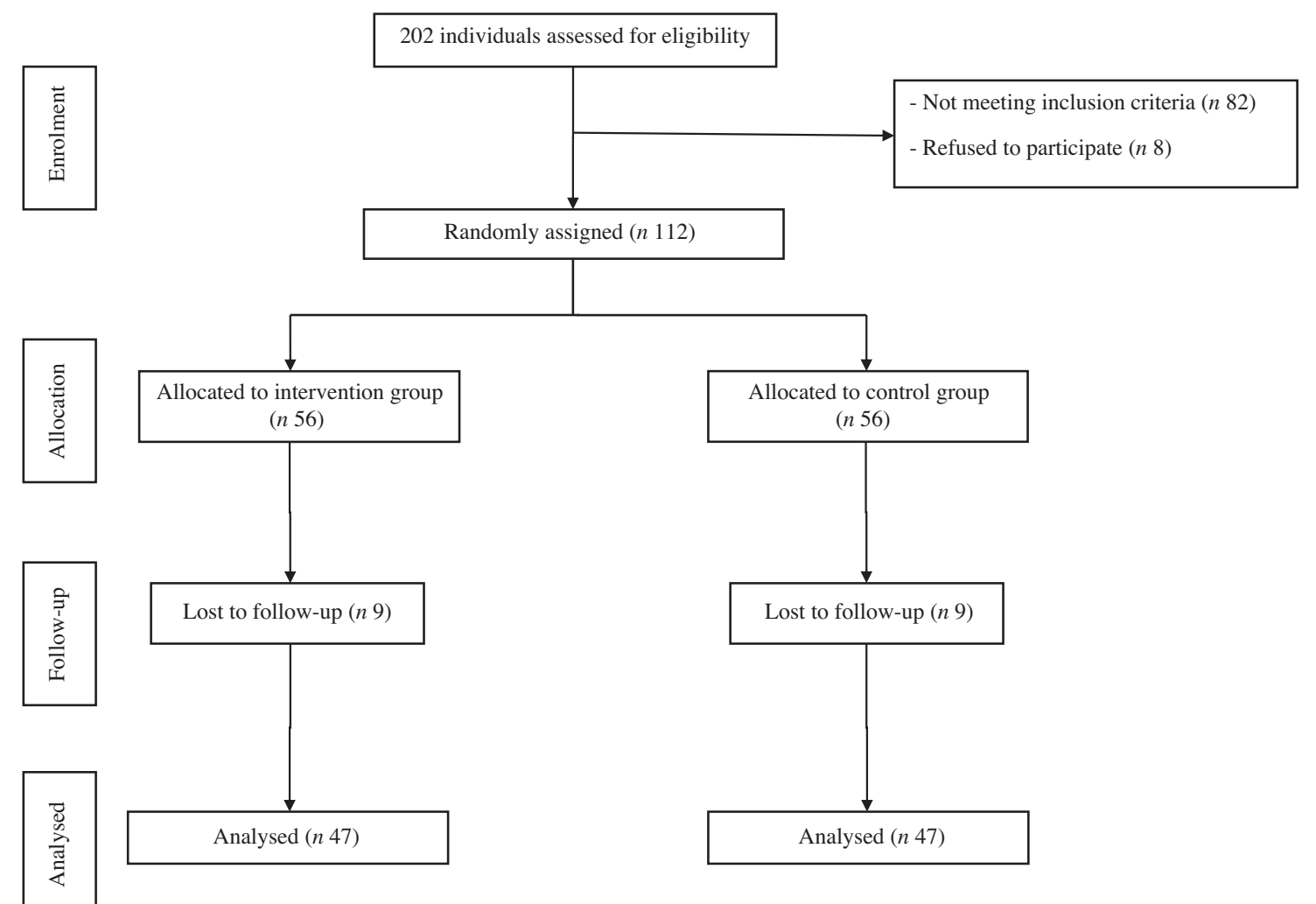

Fig. 1. Summary of patient flow diagram. 
Table 2. Dietary intakes of study participants (servings) at weeks $0,4,8$ and 12 of the study ( $n$ 47)

(Mean values and standard deviations)

\begin{tabular}{|c|c|c|c|c|c|c|c|c|c|c|c|c|c|c|c|c|c|c|c|}
\hline & \multicolumn{8}{|c|}{ Intervention group } & \multicolumn{8}{|c|}{ Control group } & \multirow{2}{*}{\multicolumn{3}{|c|}{ D* }} \\
\hline & \multicolumn{2}{|c|}{ Baseline } & \multicolumn{2}{|c|}{ Week 4} & \multicolumn{2}{|c|}{ Week 8} & \multicolumn{2}{|c|}{ Week 12} & \multicolumn{2}{|c|}{ Baseline } & \multicolumn{2}{|c|}{ Week 4} & \multicolumn{2}{|c|}{ Week 8} & \multicolumn{2}{|c|}{ Week 12} & & & \\
\hline & Mean & $\overline{S D}$ & Mean & $\overline{S D}$ & Mean & $\overline{S D}$ & Mean & $\overline{S D}$ & Mean & $\overline{S D}$ & Mean & $\overline{\mathrm{SD}}$ & Mean & $\overline{S D}$ & Mean & $\overline{S D}$ & Time & Group & Time $\times$ group \\
\hline Dairy products & 0.2 & 0.1 & 0.3 & 0.3 & 0.3 & 0.2 & 0.4 & 0.2 & 0.4 & 0.2 & 0.4 & 0.2 & 0.4 & 0.2 & 0.4 & 0.2 & 0.10 & 0.31 & 0.56 \\
\hline Vegetables & 1.5 & $1 \cdot 1$ & 1.4 & 0.9 & 1.5 & 0.8 & 1.5 & 0.8 & $2 \cdot 0$ & 1.2 & $2 \cdot 0$ & 1.2 & 2.0 & 1.0 & $2 \cdot 0$ & $1 \cdot 1$ & $<0.001$ & 0.030 & 0.52 \\
\hline Fruit & 1.8 & 1.6 & 1.8 & 1.2 & 1.6 & 1.1 & 1.8 & 1.0 & 1.2 & 0.7 & 1.6 & 0.7 & 1.7 & 1.0 & 1.9 & 0.9 & 0.004 & 0.33 & 0.36 \\
\hline Grains & $12 \cdot 6$ & 2.9 & 11.0 & $2 \cdot \overline{6}$ & 11.2 & 2.5 & 11.0 & $2 \cdot 1$ & $14 \cdot 1$ & 3.1 & $14 \cdot 1$ & $2 \cdot 8$ & $13 \cdot 6$ & 3.0 & 13.4 & 2.6 & 0.034 & $<0.001$ & 0.34 \\
\hline Whole grains & 0 & & $7 \cdot 8$ & 1.8 & $7 \cdot 7$ & $2 \cdot 3$ & $8 \cdot 1$ & $2 \cdot 3$ & 0 & & 0 & & 0 & & 0 & & 0.32 & $<0.001$ & 0.32 \\
\hline Meat & 3.5 & $1 \cdot 1$ & $3 \cdot 7$ & $1 \cdot 1$ & 3.5 & 1.3 & 3.7 & $1 \cdot 1$ & 4.4 & $1 \cdot 2$ & 4.6 & 1.0 & 4.7 & 1.2 & $4 \cdot 3$ & $1 \cdot 2$ & 0.20 & 0.011 & 0.018 \\
\hline Fat & 3.9 & 1.3 & 5.4 & 1.4 & $5 \cdot 8$ & 1.3 & $5 \cdot 6$ & $1 \cdot 1$ & 4.9 & 1.2 & 5.0 & 1.4 & 4.7 & 1.4 & 4.6 & 1.2 & $<0.001$ & $<0.001$ & 0.37 \\
\hline
\end{tabular}

* $P$ values represent the effect of time, group, and time $\times$ group interaction (computed by analysis of general linear model ANOVA for repeated measurements).

Table 3. Changes in fatty liver grade during the 12-week diet period in patients with non-alcoholic fatty liver disease in the intervention and control groups* (Numbers and percentages)

\begin{tabular}{|c|c|c|c|c|c|c|c|c|c|c|}
\hline \multirow[b]{2}{*}{ Group } & \multirow{2}{*}{$\begin{array}{c}\text { Grade of fatty } \\
\text { liver }\end{array}$} & \multicolumn{2}{|c|}{ Baseline } & \multicolumn{2}{|c|}{ Week 12} & \multirow[b]{2}{*}{ Changes: $n$} & \multirow[b]{2}{*}{$\%$} & \multicolumn{3}{|c|}{$P+$} \\
\hline & & $n$ & $\%$ & $n$ & $\%$ & & & $P_{3}$ & $P_{2}$ & $P_{1}$ \\
\hline \multirow{2}{*}{ Intervention group } & Normal & 0 & & 17 & $36 \cdot 2$ & 2-degree reduction in grade: 6 & $12 \cdot 5$ & & & \\
\hline & Grade1 & 24 & $51 \cdot 1$ & 23 & $48 \cdot 9$ & 1-degree reduction in grade: 25 & $53 \cdot 2$ & & & \\
\hline \multirow{6}{*}{ Control group } & Grade 2 & 20 & $42 \cdot 6$ & 7 & 14.9 & Without change: 15 & 31.9 & & & \\
\hline & Grade3 & 3 & $6 \cdot 4$ & 0 & 0 & 1-degree of deterioration: 1 & $2 \cdot 1$ & 0.004 & 0.003 & $<0.001$ \\
\hline & Normal & 0 & 0 & 5 & $10 \cdot 6$ & 2-degree reduction in grade: 0 & & & & \\
\hline & Grade1 & 26 & $55 \cdot 3$ & 23 & 48.9 & 1-degree reduction in grade: 12 & 25.5 & & & \\
\hline & Grade 2 & 20 & $42 \cdot 6$ & 19 & $40 \cdot 4$ & Without change: 31 & $66 \cdot 0$ & & & \\
\hline & Grade3 & 1 & $2 \cdot 1$ & 0 & 0 & 1-degree of deterioration: 14 & 8.5 & & & \\
\hline
\end{tabular}

MET, metabolic equivalent of task; WHR, waist to hip circumference ratio.

* Intervention group: baseline, $n 56$ and week 12, $n$ 47. Control group: baseline, $n 56$ and week 12, $n 47$.

$\dagger$ Based on marginal model and generalised estimating equations with a cumulative logit link function, there were significant differences between groups regarding grades of liver steatosis $(P<0.001)$. On the basis of generalised estimating equations, there were significant differences between groups in grades of liver steatosis after adjusting for baseline value of the outcome, mean change in food groups and MET value $(P=0.003)$ and after more adjusting for mean change in energy intake, weight, $\mathrm{BMI}$ and WHR $(P=0.004)$.

Table 4. Changes in liver enzymes, lipid profile, glycaemic variables and blood pressure during the 12-week diet period in patients with non-alcoholic fatty liver disease in the intervention and control groups ( $n$ 47)

(Mean values and standard deviations)

\begin{tabular}{|c|c|c|c|c|c|c|c|c|c|c|c|c|c|c|c|}
\hline & \multicolumn{6}{|c|}{ Intervention group } & \multicolumn{6}{|c|}{ Control group } & & & \\
\hline & \multicolumn{2}{|c|}{ Baseline } & \multicolumn{2}{|c|}{ Week 12} & \multicolumn{2}{|c|}{ Change } & \multicolumn{2}{|c|}{ Baseline } & \multicolumn{2}{|c|}{ Week 12} & \multicolumn{2}{|c|}{ Change } & \multicolumn{3}{|c|}{$P^{\star}$} \\
\hline & Mean & SD & Mean & SD & Mean & SD & Mean & SD & Mean & SD & Mean & SD & Time & Group & Time $\times$ group \\
\hline \multicolumn{16}{|c|}{ Serum liver enzymes (IU/I) } \\
\hline ALT & $34 \cdot 6$ & $12 \cdot 5$ & $24 \cdot 1$ & $12 \cdot 2$ & $-10.5 \dagger \dagger$ & $13 \cdot 9$ & $32 \cdot 1$ & $16 \cdot 9$ & $32 \cdot 5$ & $18 \cdot 2$ & 0.5 & $13 \cdot 3$ & 0.001 & 0.39 & $<0.001$ \\
\hline AST & $27 \cdot 7$ & $13 \cdot 6$ & 21.9 & $6 \cdot 8$ & $-5 \cdot 8 \dagger \dagger$ & $9 \cdot 6$ & $25 \cdot 5$ & 9.5 & $26 \cdot 5$ & $11 \cdot 0$ & 1.0 & 8.7 & 0.012 & 0.56 & $<0.001$ \\
\hline GGT & $26 \cdot 3$ & 11.4 & $21 \cdot 8$ & $7 \cdot 0$ & $-4.4 \dagger$ & $9 \cdot 0$ & $26 \cdot 8$ & $11 \cdot 1$ & $28 \cdot 5$ & $19 \cdot 2$ & $1 \cdot 7$ & $13 \cdot 2$ & 0.25 & 0.13 & 0.009 \\
\hline \multicolumn{16}{|l|}{ Serum lipids $(\mathrm{mg} / \mathrm{dl}) \S$} \\
\hline Total cholesterol & $192 \cdot 4$ & $40 \cdot 9$ & $174 \cdot 1$ & $37 \cdot 3$ & -18.3†† & $32 \cdot 3$ & $183 \cdot 1$ & $38 \cdot 2$ & $183 \cdot 4$ & $36 \cdot 5$ & 0.3 & $29 \cdot 0$ & 0.006 & 0.99 & 0.004 \\
\hline TAG & $167 \cdot 9$ & $13 \cdot 6$ & $156 \cdot 7$ & 11.6 & $-11 \cdot 2$ & $7 \cdot 5$ & $180 \cdot 5$ & $15 \cdot 6$ & $190 \cdot 8$ & $15 \cdot 7$ & $10 \cdot 3$ & $10 \cdot 9$ & 0.94 & 0.22 & 0.11 \\
\hline HDL-cholesterol & $41 \cdot 2$ & $7 \cdot 0$ & $43 \cdot 0$ & $5 \cdot 9$ & $1.8 \dagger$ & 4.9 & $44 \cdot 8$ & $7 \cdot 5$ & $43 \cdot 8$ & $9 \cdot 5$ & $-1 \cdot 1$ & $8 \cdot 2$ & 0.047 & 0.11 & 0.54 \\
\hline LDL-cholesterol & 114.4 & 4.5 & 101.5 & $4 \cdot 2$ & $-12 \cdot 8 \dagger$ & 4.4 & 98.9 & $4 \cdot 8$ & 99.7 & $4 \cdot 7$ & 0.9 & $3 \cdot 3$ & 0.032 & 0.14 & 0.014 \\
\hline \multicolumn{16}{|l|}{ Glycaemic variables } \\
\hline FBS $(m g / d l) \S$ & $88 \cdot 1$ & $10 \cdot 2$ & $86 \cdot 9$ & $8 \cdot 2$ & $-1 \cdot 2$ & $9 \cdot 5$ & $87 \cdot 5$ & $10 \cdot 0$ & 88.9 & $10 \cdot 9$ & 1.4 & $10 \cdot 6$ & 0.91 & 0.70 & 0.20 \\
\hline Serum insulin (mU/l) & $17 \cdot 0$ & $9 \cdot 3$ & $14 \cdot 9$ & $7 \cdot 9$ & $-2 \cdot 1 \dagger$ & $6 \cdot 4$ & $14 \cdot 7$ & $7 \cdot 1$ & $14 \cdot 5$ & $7 \cdot 9$ & -0.3 & $5 \cdot 7$ & 0.63 & 0.39 & $0 \cdot 15$ \\
\hline HOMA-IR & 3.5 & 0.3 & $3 \cdot 2$ & $0 \cdot 3$ & $-0.5 \dagger$ & $0 \cdot 2$ & $3 \cdot 2$ & $0 \cdot 2$ & $3 \cdot 1$ & $0 \cdot 2$ & -0.03 & $0 \cdot 2$ & $0 \cdot 10$ & 0.35 & $0 \cdot 16$ \\
\hline QUICKI & 0.3 & 0.02 & 0.3 & 0.02 & 0.007 & 0.02 & 0.3 & 0.02 & 0.3 & 0.02 & 0.007 & 0.02 & 0.70 & 0.41 & 0.25 \\
\hline \multicolumn{16}{|l|}{ Blood pressure (mmHg) } \\
\hline SBP & $125 \cdot 5$ & $7 \cdot 4$ & $122 \cdot 3$ & $5 \cdot 5$ & $-3 \cdot 2$ & $5 \cdot 5$ & $125 \cdot 1$ & $8 \cdot 3$ & $125 \cdot 5$ & $7 \cdot 1$ & 0.4 & $6 \cdot 2$ & 0.026 & 0.31 & 0.004 \\
\hline DBP & $79 \cdot 7$ & $4 \cdot 7$ & $78 \cdot 0$ & $5 \cdot 0$ & $-1 \cdot 7$ & $4 \cdot 3$ & 79.9 & $5 \cdot 6$ & $80 \cdot 7$ & 4.4 & 0.8 & $4 \cdot 8$ & 0.37 & 0.10 & 0.008 \\
\hline
\end{tabular}

ALT, alanine aminotransferase; AST, aspartate aminotransferase; GGT, $\gamma$-glutamyltransferase; FBS, fasting blood sugar; HOMA-IR, homoeostasis model of assessment-estimated insulin resistance; QUICKI, quantitative insulin sensitivity check index; SBP, systolic blood pressure; DBP, diastolic blood pressure.

* $P$ values represent the effect of time, group and time $\times$ group interaction (computed by analysis of general linear model ANOVA for repeated measurements).

$P$ values were computed by paired-samples $t$ test: $\dagger P<0.05, \dagger \dagger P<0.001$.

$\ddagger$ On the basis of independent-samples $t$ test, there were no significant differences between groups with regard to baseline values $(P>0.05)$

$\S$ To convert cholesterol in $\mathrm{mg} / \mathrm{dl}$ to $\mathrm{mmol} / \mathrm{l}$, multiply by 0.0259 . To convert TAG in $\mathrm{mg} / \mathrm{dl}$ to $\mathrm{mmol} / \mathrm{l}$, multiply by 0.0113 . To convert FBS in mg/dl to $\mathrm{mmol} / \mathrm{l}$, multiply by 0.0555 . 
Table 5. Adjusted changes in liver enzymes, lipid profile, glycaemic variables and blood pressure during the 12-week diet period in patients with non-alcoholic fatty liver disease in the intervention and control groups ( $n$ 47) (Mean values and standard deviations)

\begin{tabular}{|c|c|c|c|c|c|}
\hline & \multicolumn{2}{|c|}{ Intervention group } & \multicolumn{2}{|c|}{ Control group } & \multirow[b]{2}{*}{$P^{\star}$} \\
\hline & Mean & SD & Mean & SD & \\
\hline \multicolumn{6}{|l|}{ ALT (IU/I) } \\
\hline Model 1† & -8.5 & $2 \cdot 0$ & -1.5 & $2 \cdot 1$ & 0.022 \\
\hline Model $2 \ddagger$ & -9.0 & 1.9 & -1.0 & 1.9 & 0.012 \\
\hline \multicolumn{6}{|l|}{ AST (IU/I) } \\
\hline Model 1 & $-4 \cdot 8$ & 1.4 & 0.01 & 1.4 & 0.019 \\
\hline Model 2 & $-5 \cdot 2$ & 1.4 & 0.3 & 1.4 & 0.010 \\
\hline \multicolumn{6}{|l|}{ GGT (IU/I) } \\
\hline Model 1 & $-5 \cdot 9$ & $2 \cdot 0$ & $3 \cdot 1$ & $2 \cdot 0$ & 0.006 \\
\hline Model 2 & $-5 \cdot 6$ & $2 \cdot 1$ & $2 \cdot 9$ & $2 \cdot 1$ & 0.012 \\
\hline \multicolumn{6}{|c|}{ Total cholesterol $(\mathrm{mg} / \mathrm{dl}) \S$} \\
\hline Model 1 & $-15 \cdot 2$ & 4.5 & $-2 \cdot 7$ & 4.5 & 0.11 \\
\hline Model 2 & $-15 \cdot 9$ & $5 \cdot 1$ & $-2 \cdot 1$ & $5 \cdot 0$ & 0.09 \\
\hline \multicolumn{6}{|c|}{ TAG $(\mathrm{mg} / \mathrm{dl}) \S$} \\
\hline Model 1 & $-5 \cdot 6$ & 1.3 & 4.7 & $1 \cdot 2$ & 0.53 \\
\hline Model 2 & $-6 \cdot 5$ & $1 \cdot 1$ & 5.6 & 1.1 & 0.47 \\
\hline \multicolumn{6}{|c|}{ HDL-cholesterol $(\mathrm{mg} / \mathrm{dl}) \S$} \\
\hline Model 1 & $-2 \cdot 3$ & 1.4 & -0.5 & 1.4 & 0.32 \\
\hline Model 2 & $-2 \cdot 5$ & 1.4 & -0.3 & 1.4 & 0.24 \\
\hline \multicolumn{6}{|c|}{ LDL-cholesterol $(\mathrm{mg} / \mathrm{dll}) \S$} \\
\hline Model 1 & -9.8 & $4 \cdot 3$ & $-2 \cdot 2$ & $4 \cdot 3$ & 0.25 \\
\hline Model 2 & $-10 \cdot 0$ & $4 \cdot 3$ & -1.9 & $4 \cdot 3$ & 0.25 \\
\hline \multicolumn{6}{|l|}{ FBS $(\mathrm{mg} / \mathrm{dll}) \S$} \\
\hline Model 1 & -0.9 & 1.6 & $1 \cdot 2$ & 1.5 & 0.35 \\
\hline Model 2 & $-0 \cdot 1$ & 1.6 & 1.2 & 1.6 & 0.36 \\
\hline \multicolumn{6}{|c|}{ Serum insulin $(\mathrm{mU} / \mathrm{l})$} \\
\hline Model 1 & -1.4 & 0.9 & -0.9 & 0.9 & 0.79 \\
\hline Model 2 & -1.4 & 1.0 & $-1 \cdot 0$ & $1 \cdot 0$ & 0.79 \\
\hline \multicolumn{6}{|l|}{ HOMA-IR } \\
\hline Model 1 & -0.3 & 0.3 & -0.2 & 0.3 & 0.76 \\
\hline Model 2 & $-0 \cdot 3$ & 0.3 & -0.2 & 0.3 & 0.75 \\
\hline \multicolumn{6}{|l|}{ QUICKI } \\
\hline Model 1 & -0.02 & 0.01 & -0.02 & 0.01 & 0.86 \\
\hline Model 2 & -0.02 & 0.01 & -0.02 & 0.01 & 0.86 \\
\hline \multicolumn{6}{|l|}{$\mathrm{SBP}(\mathrm{mmHg})$} \\
\hline Model 1 & $-3 \cdot 4$ & 0.9 & 0.7 & 0.9 & 0.004 \\
\hline Model 2 & $-4 \cdot 0$ & 0.9 & $1 \cdot 2$ & 0.9 & $>0.001$ \\
\hline \multicolumn{6}{|l|}{ DBP $(\mathrm{mmHg})$} \\
\hline Model 1 & $-1 \cdot 6$ & 0.8 & $1 \cdot 1$ & 0.8 & 0.007 \\
\hline Model 2 & -1.9 & 0.8 & $1 \cdot 1$ & 0.8 & 0.012 \\
\hline
\end{tabular}

ALT, alanine aminotransferase; AST, aspartate aminotransferase; GGT, $\gamma$-glutamyltransferase; FBS, fasting blood sugar; HOMA-IR, homoeostasis model of assessment-estimated insulin resistance; QUICKI, quantitative insulin sensitivity check index; SBP, systolic blood pressure; DBP, diastolic blood pressure; MET, metabolic equivalent of task; WHR, waist to hip circumference ratio.

* $P$ values were computed by univariate ANCOVA.

† Adjusted for baseline value of the outcome, mean change in food groups and MET value.

$\ddagger$ Adjusted for baseline value of the outcome, mean change in food groups, energy intake, weight, BMI, WHR and MET value.

$\S$ To convert cholesterol in $\mathrm{mg} / \mathrm{dl}$ to $\mathrm{mmol} / \mathrm{l}$, multiply by 0.0259 . To convert TAG in $\mathrm{mg} / \mathrm{dl}$ to $\mathrm{mmol} / \mathrm{l}$, multiply by 0.0113 . To convert FBS in $\mathrm{mg} / \mathrm{dl}$ to $\mathrm{mmol} / \mathrm{l}$, multiply by 0.0555 .

different between the two groups. The intervention group showed a greater reduction in these parameters than did the control group. In addition, no significant differences were found between the two groups in terms of serum concentrations of TAG, HDL-cholesterol, insulin and fasting blood sugar and homeostatic model assessment of insulin resistance, weight, BMI or WHR.

After adjusting for baseline value of the outcome, mean changes in food groups and MET value and after adjusting more for mean changes in energy intake, weight, BMI and WHR, the differences in changes of serum, total cholesterol and LDLcholesterol concentrations between the two groups became non-significant. Moreover, other findings were not significantly changed after the adjustments were made (Table 4).

\section{Discussion}

NAFLD poses substantial challenges to public health across the globe. Therefore, to improve NAFLD risk factors, researchers have become interested in the effectiveness of different dietary patterns. Consequently, in the present study, it is hypothesised that patients with NAFLD who included whole-grain foods in their diet would show a more considerable improvement in laboratory liver tests.

In the present study, we showed that the consumption of whole grains for 12 weeks led to statistically and clinically significant improvements in grade of steatosis and liver enzymes. Significant differences in hepatic steatosis and liver enzymes were observed between the groups, even after adjustment with covariates. This indicates that whole grain by itself and 
independent of weight loss improved the above outcomes. Data on the effects of whole grains on hepatic steatosis and liver enzymes among NAFLD patients, however, are scarce. Results from a case-control study with thirty-four non-alcoholic steatohepatitis patients suggested that whole grains were potentially useful for improving hepatic steatosis and liver enzymes ${ }^{(12)}$. Also, in a study by Abdelmalek et al. ${ }^{(25)}$, it was found that betaine, as a beneficial factor in whole grain, improved hepatic steatosis. Tovar et al. ${ }^{(15)}$ observed a significant decrease in $\gamma$-glutamyltransferase concentrations after consumption of whole-grain barley product supplementation, compared with a control diet, in overweight women. The results of study conducted by Angelino et al. ${ }^{(14)}$, however, showed that whole-grain consumption for 12 weeks had no effect on liver enzymes. The discrepancy was probably due to an approximate doubling in total daily fibre intake in the study by Tovar et al. ${ }^{(15)}$. This efficacy can at least be partially attributed to the effects of the whole grains on modulation of the microbiota composition in humans, with corresponding changes to gut microflora metabolites including $\mathrm{SCFA}^{(26)}$.

At an anatomical location, the liver is directly supplied with blood flow from the intestine. As a result, changes in intestinal permeability caused by dysbiosis gut microbiota allow the translocation of bacterial products into the portal vein and development of obesity-related metabolic diseases such as NAFLD. The portion of gut microbiota in the progression of NAFLD is mainly based on increased hepatic reactive oxygen species due to the increased pro-inflammatory markers in the intestinal lumen. Some studies have shown associations between wholegrain consumption and inflammatory markers ${ }^{(27-30)}$. It is assumed in our study that the reduction in oxidative stress and inflammatory markers due to the consumption of whole grains has been responsible for the decrease in liver enzymes and steatosis; nonetheless, further investigations are needed. On the other hand, whole-grain foods are a good source of micronutrients such as $\mathrm{Cu}$, which is identified as a key player in various metabolic disorders ${ }^{(31)}$. Reduced serum and hepatic $\mathrm{Cu}$ concentrations characterise NAFLD patients and seem to have an important role in the progression of metabolic diseases.

Moreover, findings from the present study showed that the consumption of whole grains did not affect serum fasting blood sugar and insulin concentrations. In a report from the Framingham Offspring Cohort Study, the number of daily servings of whole-grain foods was inversely related to insulin resistance and fasting insulin concentrations ${ }^{(13)}$. In the Insulin Resistance Atherosclerosis Study, a greater intake of whole grains was associated with higher insulin sensitivity ${ }^{(32)}$. Similar results were observed in a cross-sectional study of 285 adolescents $^{(33)}$. This discrepancy might be due to the number of daily servings of whole-grain foods, nature of the disease and duration of intervention.

Whole-grain foods are thought to have a beneficial effect on glycaemic variables due to their greater fibre content, which leads to a slower or reduced digestion of macronutrients, reduced blood glucose burden and lower insulin concentrations when supplemented in clinical trials ${ }^{(34)}$. Also, bioactive compounds in whole-grain foods, such as phenolic acids and alkylresorcinols, seem to contribute to the antioxidant and anti-inflammatory effects ${ }^{(29)}$.

The lipid profile results of the present analyses are in line with the Framingham Offspring Cohort Study that showed whole-grain foods were related to lower LDL-cholesterol and total cholesterol concentrations and not to HDL-cholesterol or TAG concentrations ${ }^{(14)}$. The Tehran, Lipid and Glucose Study with 827 men and women revealed that intake of traditional Iranian whole-grain foods was associated with lower TAG concentrations but not with any other lipid variables ${ }^{(11)}$. Similarly, an improvement in the lipid profiles has also been found in overweight or obese subjects, after consumption of oat-based products for 12 weeks $^{(35)}$ and in subjects with intake of $\beta$-glucan-rich products for 8 weeks ${ }^{(15)}$. The beneficial effects of whole-grain foods have been, at least partly, ascribed to their lipid-lowering properties, particularly for whole grains containing relatively high amounts of viscous soluble fibre ${ }^{(36)}$.

In the present study, we found a $3 \mathrm{mmHg}$ reduction in systolic blood pressure and a $1 \mathrm{mmHg}$ reduction in diastolic blood pressure. These results are also consistent with results of previous studies that reported the beneficial effects of whole grains on blood pressure ${ }^{(37,38)}$. Contrary to our study, Angelino et $a l .{ }^{(14)}$ found no significant variations in systolic and diastolic blood pressure, probably because of low consumption of daily whole grains. The beneficial effects of whole grains on blood pressure could be explained by potential bioactivity of micronutrients such as $\mathrm{Ca}, \mathrm{Mg}, \mathrm{Zn}, \mathrm{K}$ and vitamin $\mathrm{D}^{(31)}$.

Based on an assessment of dietary intake at baseline and every 4 weeks, participants in the intervention group had a greater reduction in energy intake than did participants in the control group. This might be explained by the fact that whole grains could reduce appetite; however, further investigations are required. Participants in the intervention group significantly improved their diet quality by increasing their intake of dietary fibres to almost $14 \mathrm{~g} / 4184 \mathrm{~kJ}$, which is the amount recommended in the 2012 dietary guidelines for Americans ${ }^{(9)}$. Participants in the whole-grain group also increased their intake of beneficial micronutrients which potentially contribute to the management of NAFLD ${ }^{(31)}$. Thus, the consumption of whole-grain foods would be expected to benefit metabolic diseases, such as obesity, type 2 diabetes and NAFLD.

The strength of the present study rests on its high completion and compliance rate. Furthermore, consumption of whole-grain food is a low-cost management strategy for NAFLD patients.

A limitation of the present study is its short duration of interventions.

In conclusion, this open-label randomised controlled clinical trial provided some evidence that consumption of whole-grain foods improved hepatic features in patients with NAFLD. Nevertheless, future clinical studies examining larger sample sizes for longer periods are required to determine the long-term health benefits of whole grains.

\section{Acknowledgements}

The authors would like to express their gratitude towards the Urmia University of Medical Sciences, for the facilities and financial support. The authors would also like to thank the 
patients who participated in the present study. This article is based on data obtained from a master's thesis in Nutrition.

The present study was supported by Urmia University of Medical Sciences. This trial was registered at the Iranian Registry of Clinical Trials website as IRCT20170206032417N3.

The authors' responsibilities were as follows: M. A. and M. D. conceived and designed the study and analysed the data; J. A. provided material and technical support; F. B. wrote the manuscript; M. A. critically revised the manuscript for important intellectual content and M. A. had primary responsibility. All authors read and approved the final manuscript.

There are no conflicts of interest.

The lead author affirms that this manuscript is an honest, accurate, and transparent account of the study being reported. The reporting of this work is compliant with CONSORT guidelines. The lead author affirms that no important aspects of the study have been omitted and that any discrepancies from the study as planned have been explained.

\section{References}

1. Loomba R \& Sanyal AJ (2013) The global NAFLD epidemic. Nat Rev Gastroenterol Hepatol 10, 686-690.

2. Moghaddasifar I, Lankarani K, Moosazadeh M, et al. (2016) Prevalence of non-alcoholic fatty liver disease and its related factors in Iran. Int J Organ Transplant Med 7, 149.

3. Fleischman MW, Budoff M, Ifran Zeb DL, et al. (2014) NAFLD prevalence differs among Hispanic subgroups: the Multi-Ethnic Study of Atherosclerosis. World J Gastroenterol 20, 4987.

4. Chalasani N, Younossi Z, Lavine JE, et al. (2012) The diagnosis and management of non-alcoholic fatty liver disease: practice Guideline by the American Association for the Study of Liver Diseases, American College of Gastroenterology, and the American Gastroenterological Association. Hepatology 55, 2005-2023.

5. Tsochatzis E, Papatheodoridis GV, Manesis EK, et al. (2008) Metabolic syndrome is associated with severe fibrosis in chronic viral hepatitis and non-alcoholic steatohepatitis. Aliment Pharmacol Ther 27, 80-89.

6. Gao X \& Fan JG (2013) Diagnosis and management of nonalcoholic fatty liver disease and related metabolic disorders: consensus statement from the Study Group of Liver and Metabolism, Chinese Society of Endocrinology. J Diabetes $\mathbf{5}$, 406-415.

7. Rabl C \& Campos GM (2012) The impact of bariatric surgery on nonalcoholic steatohepatitis. Semin Liver Dis 32, 80-91.

8. US Department of Health and Human Services and US Department of Agriculture (2015) 2015-2020 Dietary Guidelines for Americans. 8th Edition. http://health.gov/dietaryguidelines/ 2015/guidelines/ (accessed April 2016).

9. US Department of Health and Human Services (2012) Dietary guidelines for Americans. http://www.dietaryguidelines.gov (accessed November 2012).

10. Anderson JW \& Hanna TJ (1999) Impact of nondigestible carbohydrates on serum lipoproteins and risk for cardiovascular disease. J Nutr 129, 1457S-1466S.

11. Esmaillzadeh A, Mirmiran P \& Azizi F (2005). Whole-grain consumption and the metabolic syndrome: a favorable association in Tehranian adults. Eur J Clin Nutr 59, 353.

12. Georgoulis M, Kontogianni MD, Tileli N, et al. (2014) The impact of cereal grain consumption on the development and severity of non-alcoholic fatty liver disease. Eur J Nutr 53, $1727-1735$.
13. McKeown NM, Meigs JB, Liu S, et al. (2002). Whole-grain intake is favorably associated with metabolic risk factors for type 2 diabetes and cardiovascular disease in the Framingham Offspring Study. Am J Clin Nutr 76, 390-398.

14. Angelino D, Martina A, Rosi A, et al. (2019) Glucose-and lipid-related biomarkers are affected in healthy obese or hyperglycemic adults consuming a whole-grain pasta enriched in prebiotics and probiotics: a 12 -week randomized controlled trial. J Nutr 149, 1714-1723.

15. Tovar J, Nilsson A, Johansson M, et al. (2014) Combining functional features of whole-grain barley and legumes for dietary reduction of cardiometabolic risk: a randomised cross-over intervention in mature women. Br J Nutr 111, 706-714.

16. Cooper D, Kable M, Marco M, et al. (2017) The effects of moderate whole grain consumption on fasting glucose and lipids, gastrointestinal symptoms, and microbiota. Nutrients 9, 173.

17. Kirwan JP, Malin SK, Scelsi AR, et al. (2016) A whole-grain diet reduces cardiovascular risk factors in overweight and obese adults: a randomized controlled trial. J Nutr 146, 2244-2251.

18. Sandberg JC, Björck IM \& Nilsson, AC (2017) Effects of whole grain rye, with and without resistant starch type 2 supplementation, on glucose tolerance, gut hormones, inflammation and appetite regulation in an 11-14.5 hour perspective; a randomized controlled study in healthy subjects. Nutr J 16, 25.

19. Malin SK, Kullman EL, Scelsi AR, et al. (2018). A whole-grain diet reduces peripheral insulin resistance and improves glucose kinetics in obese adults: a randomized-controlled trial. Metabolism 82, 111-117.

20. Kurtz AB, Dubbins PA, Rubin CS, et al. (1981) Echogenicity: analysis, significance, and masking. AJR Am J Roentgenol 137, 471-476.

21. Sharma S \& Fleming SE (2012) Use of HbA(1C) testing to diagnose pre-diabetes in high risk African American children: a comparison with fasting glucose and HOMA-IR. Diabetes Metab Syndr 6, 157-162.

22. World Health Organization \& Warsaw-Nutrition Unit (1989) Measuring Obesity-classification and Description of Anthropometric Data: report on a WHO Consultation on the Epidemiology of Obesity, Warsaw, 21-23 October 1987. Copenhagen: World Health Organization, Regional Office for Europe.

23. Ainsworth BE, Haskell WL, Whitt MC, et al. (2000) Compendium of physical activities: an update of activity codes and MET intensities. Med Sci Sports Exerc 32, Suppl. 1, S498-S504.

24. Katcher HI, Legro RS, Kunselman AR, et al. (2008) The effects of a whole grain-enriched hypocaloric diet on cardiovascular disease risk factors in men and women with metabolic syndrome. Am J Clin Nutr 87, 79-90.

25. Abdelmalek MF, Sanderson SO, Angulo P, et al. (2009). Betaine for nonalcoholic fatty liver disease: results of a randomized placebo-controlled trial. Hepatology 50, 1818-1826.

26. Martínez I, Lattimer JM, Hubach KL, et al. (2013) Gut microbiome composition is linked to whole grain-induced immunological improvements. ISME J 7, 269.

27. Hajihashemi P, Azadbakht L, Hashemipor M, et al. (2014) Whole-grain intake favorably affects markers of systemic inflammation in obese children: a randomized controlled crossover clinical trial. Mol Nutr Food Res 58, 1301-1308.

28. Vitaglione P, Mennella I, Ferracane R, et al. (2014). Whole-grain wheat consumption reduces inflammation in a randomized controlled trial on overweight and obese subjects with unhealthy dietary and lifestyle behaviors: role of polyphenols bound to cereal dietary fiber. Am J Clin Nutr 101, 251-261. 
29. Lutsey PL, Jacobs DR, Kori S, et al. (2007) Whole grain intake and its cross-sectional association with obesity, insulin resistance, inflammation, diabetes and subclinical CVD: the MESA Study. Br J Nutr 98, 397-405.

30. Qi L, Van Dam RM, Liu S, et al. (2006) Whole-grain, bran, and cereal fiber intakes and markers of systemic inflammation in diabetic women. Diabetes Care 29, 207-211.

31. Tarantino G, Porcu C, Arciello M, et al. (2018) Prediction of carotid intima-media thickness in obese patients with low prevalence of comorbidities by serum copper bioavailability. J Gastroenterol Hepatol 33, 1511-1517.

32. Liese AD, Roach AK, Sparks KC, et al. (2003). Whole-grain intake and insulin sensitivity: the Insulin Resistance Atherosclerosis Study. Am J Clin Nutr 78, 965-971.

33. Steffen LM, Jacobs Jr DR, Murtaugh MA, et al. (2003). Whole grain intake is associated with lower body mass and greater insulin sensitivity among adolescents. Am J Epidemiol 158, $243-250$
34. Jensen MK, Koh-Banerjee P, Franz M, et al. (2006). Whole grains, bran, and germ in relation to homocysteine and markers of glycemic control, lipids, and inflammation. Am J Clin Nutr 83, 275-283.

35. Davy BM, Davy KP, Ho RC, et al. (2002) High-fiber oat cereal compared with wheat cereal consumption favorably alters LDL-cholesterol subclass and particle numbers in middle-aged and older men. Am J Clin Nutr 76, 351-358.

36. Ross AB, Godin J-P, Minehira K, et al. (2013). Increasing whole grain intake as part of prevention and treatment of nonalcoholic fatty liver disease. Int J Endocrinol 2013, 585876.

37. Tighe P, Duthie G, Vaughan N, et al. (2010). Effect of increased consumption of whole-grain foods on blood pressure and other cardiovascular risk markers in healthy middle-aged persons: a randomized controlled trial. Am J Clin Nutr 92, 733-740.

38. Streppel MT, Arends LR, van't Veer P, et al. (2005). Dietary fiber and blood pressure: a meta-analysis of randomized placebo-controlled trials. Arch Intern Med 165, 150-156. 